\title{
An Inverted FL Antenna for Dual-Frequency Operation
}

Hisamatsu Nakano, Fellow, IEEE, Yusuke Sato, Hiroaki Mimaki, Member, IEEE, and Junji Yamauchi, Member, IEEE

\begin{abstract}
An inverted FL antenna (InvFLA) is analyzed to obtain dual-frequency operation at 2.45 and $5.2 \mathrm{GHz}$ (wireless LAN system frequencies). The InvFLA is composed of inverted FL elements, a parasitic element, and a ground plate, where these lie in the same plane, i.e., the structure is a card-type structure having a co-planar ground plate. The antenna height above the ground plate is very small: $5.5 \mathrm{~mm}=0.045$ wavelength at $2.45 \mathrm{GHz}$. The analysis shows that the InvFLA has a $4.1 \%$ bandwidth around 2.45 GHz and a $31.8 \%$ bandwidth around $5.2 \mathrm{GHz}$, both for a VSWR $=2$ criterion. The gain is calculated to be $0.9 \mathrm{dBi}$ at $2.45 \mathrm{GHz}$ and $1.7 \mathrm{dBi}$ at $5.2 \mathrm{GHz}$, with a small gain variation in each of the VSWR bands.
\end{abstract}

Index Terms-Card-type antenna, dual-frequency operation, finite-difference time-domain (FDTD) analysis, inverted F, inverted L.

\section{INTRODUCTION}

$\mathbf{T}$ HE increasing demand for wireless communications has been accelerating development of new antennas that operate in the required frequency bands [1]-[5]. The dual-frequency antenna is one of these new antennas, and so far numerous efforts have been made in this area [6]-[8]. For example, Wong has investigated microstrip patches for dual-frequency operation and summarized them in [9].

This paper presents an antenna that responds to the abovementioned trend: an inverted FL antenna (InvFLA) for dualfrequency operation. The InvFLA is made of a thin conducting film, having a flat structure, as shown in Fig. 1(b), where both the radiation element (inverted $F$ and $L$ strip lines) in the positive $y$ space and the ground plate (GP) in the negative $y$ space lie in the same plane $(x-y$ plane). In other words, the InvFLA has a co-planar ground plate, forming a card-type antenna structure.

The card-type InvFLA structure differs from the layered microstrip antenna structure for dual-frequency operation in [9], where the ground plate backs a radiation element (patch element), i.e., the patch is parallel to the ground plate. It is emphasized that the card-type structure facilitates the use of the InvFLA in PC card devices for personal computers or inside mobile phone handsets.

After a brief summary of the analysis methods, which are based on the finite-difference time-domain method (FDTDM) [10], this paper investigates an InvFLA for realizing dual-frequency operation at 2.45 and $5.2 \mathrm{GHz}$ (frequencies used for wireless LAN communications). Note that the final structural parameters for the InvFLA are obtained through a step-by-step

Manuscript received December 14, 2004; revised May 22, 2005.

The authors are with the College of Engineering, Hosei University, Tokyo 184-8584, Japan (e-mail: nakano@k.hosei.ac.jp).

Digital Object Identifier 10.1109/TAP.2005.852502

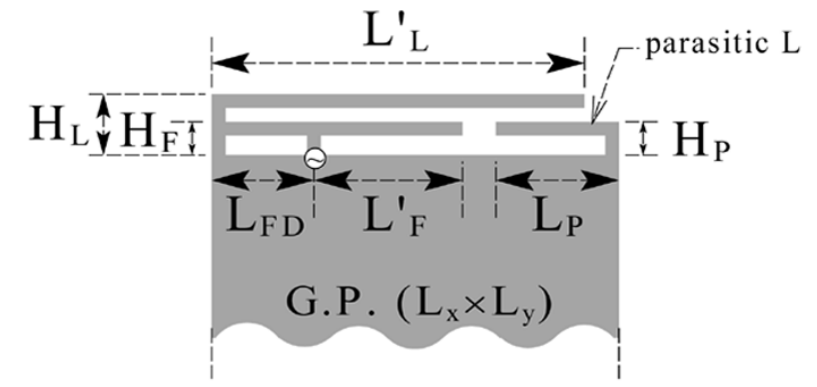

(a)

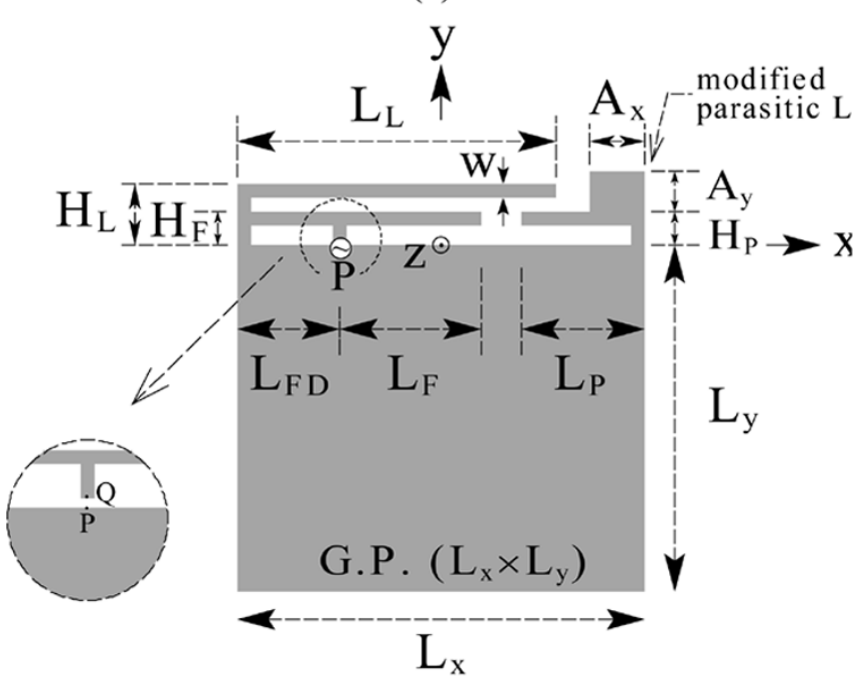

(b)

Fig. 1. Antenna structures. (a) A compound of inverted $L$ and $F$ elements (referred to as a compounded $L F$ ) with a parasitic $L$ element above a co-planar ground plate (GP). (b) A compounded LF with a modified parasitic $L$ element above a co-planar ground plate, referred to as the inverted FL antenna (InvFLA). The ground plate size in (a) is the same as that in (b): $L_{x} \times L_{y}=30 \mathrm{~mm} \times$ $25.5 \mathrm{~mm}$.

investigation of the following structures: 1 ) an inverted $L$ element; 2) an inverted $F$ element; 3 ) a compound of the inverted $L$ and $F$ elements, referred to as a compounded $L F ; 4)$ the compounded $L F$ with a parasitic inverted $L$ element; and 5) the compounded LF with a modified parasitic inverted $L$ element. Also, note that the design process presented in this paper is not necessarily restricted to the specific frequencies 2.45 and $5.2 \mathrm{GHz}$. It is possible to apply the same design technique to other dual-frequency antenna designs.

For confirmation of the FDTDM results (obtained using the FDTDM computer programs developed by the authors), experimental results are presented. A good agreement between the FDTDM results and the experimental results is found. 


\section{CONFIGURATION}

Fig. 1(a) shows a step involved in reaching the antenna structure of Fig. 1(b). The radiation element in Fig. 1(a) is a compound of three sub-elements: an inverted $L$ element (see inset (I) of Fig. 2, where elements $\alpha-\beta-\gamma$ and $\alpha^{\prime}-\beta^{\prime}-\gamma^{\prime}$ are collectively referred to as the "inverted $L$ element"), an inverted $F$ element (see inset (II) of Fig. 2), and a parasitic inverted $L$ element (simply referred to as a parasitic $L$ element).

Fig. 1(b) is a modified version of the structure in Fig. 1(a), where a small protrusion of area $A_{x} \times A_{y}$ is added to the parasitic $L$ element in Fig. 1(a). The structure of Fig. 1(b) is based on the inverted $L$ and $F$ elements, and hence it is referred to as the inverted FL antenna (InvFLA). It is emphasized that the InvFLA is made of a thin conducting film, where the ground plate (GP) is a co-planar ground plate, i.e., the ground plate and the radiation element lie in the same plane ( $x-y$ plane), forming a card-type structure.

The heights of the radiation sub-elements $\left(H_{L}, H_{F}\right.$, and $\left.H_{P}\right)$ and the ground plate size $\left(L_{x} \times L_{y}\right)$ in Fig. 1(b) are the same as those in Fig. 1(a). However, the horizontal lengths $L_{L}$ and $L_{F}$ in Fig. 1(b) are slightly different from $L_{L}^{\prime}$ and $L_{F}^{\prime}$ in Fig. 1(a), respectively, as will be revealed later.

The InvFLA is excited at terminals $\mathrm{P}$ and $\mathrm{Q}$, where the distance between $\mathrm{P}$ and $\mathrm{Q}$ is fixed to be $0.5 \mathrm{~mm}$. The distance from the left side edge of the ground plate to terminal $\mathrm{P}$ is denoted as $L_{\mathrm{FD}}$. For the experimental work, the InvFLA is excited through a 50-ohm coaxial line without a balun circuit, where the inner conductor of the coaxial line is connected to point $\mathrm{Q}$ and the outer conductor is soldered to the ground plate. To facilitate a PC card implementation, a thin coaxial line can be used (a coaxial line whose outer diameter is $0.8 \mathrm{~mm}$ is commercially available).

The ground plate size and the strip line width of the radiation element are fixed to be $L_{x} \times L_{y}=30 \mathrm{~mm} \times 25.5 \mathrm{~mm}$ and $w=1 \mathrm{~mm}$, respectively, throughout this paper. There are nine structural parameters to be determined: the heights $\left(H_{L}\right.$, $\left.H_{F}, H_{P}\right)$, the strip line lengths $\left(L_{L}, L_{F}, L_{P}\right)$, the protrusion size $\left(A_{x}, A_{y}\right)$, and the feed point location $L_{\mathrm{FD}}$. In this paper, the heights $\left(H_{L}, H_{F}, H_{P}\right)$ are pre-selected to be small with respect to the wavelengths at 2.45 and $5.2 \mathrm{GHz}:\left(H_{L}, H_{F}, H_{P}\right)=$ (4.5 mm, $2.5 \mathrm{~mm}, 2.5 \mathrm{~mm})$. The remaining structural parameters $\left(L_{L}, L_{F}, L_{P}\right),\left(A_{x}, A_{y}\right)$ and $L_{\mathrm{FD}}$ are to be determined for operation at 2.45 and $5.2 \mathrm{GHz}$.

\section{ANALYSIS AND DISCUSSION}

Analysis is performed using the finite-difference time-domain method (FDTDM). For this, Yee's algorithm based on rectangular cells [10] is adopted, where the analysis space is terminated using Liao's second order absorbing boundary condition [11]. The antenna excitation is modeled by a delta-gap voltage source $V_{\text {in }}(t)$, which is defined by a sine function modulated by a Gaussian function: $V_{\text {in }}(t)=V_{\text {gauss }}(t) \sin \omega t$, where $V_{\text {gauss }}(t)=\exp \{-(t-T) / K T\}^{2}$, with $K=0.29$ and $T=0.646 / f_{3 \mathrm{~dB}}$. Note that $f_{3 \mathrm{~dB}}$ is the frequency at which the power spectrum (|Fourier transform of $\left.V_{\text {gauss }}(t)\right|^{2}$ ) drops $3 \mathrm{~dB}$ from its maximum value. The electric field at a far-field point, $E_{\text {Far }}$ (composed of $E_{\theta}$ and $E_{\phi}$ ), is calculated on the basis of the equivalence principle [12].

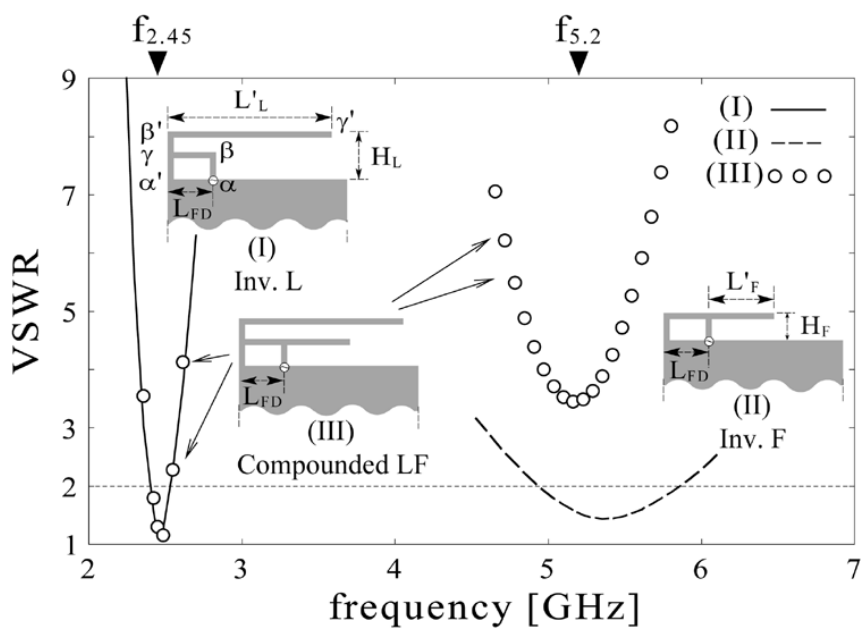

Fig. 2. VSWRs of three structures. The structural parameters for an inverted $L$ element (I) are $\left(H_{L}, L_{L}^{\prime}\right)=(4.5 \mathrm{~mm}, 27.5 \mathrm{~mm})$ and $L_{\mathrm{FD}}=7.5 \mathrm{~mm}$, and those for an inverted $F$ element (II) are $\left(H_{F}, L_{F}^{\prime}\right)=(2.5 \mathrm{~mm}, 11 \mathrm{~mm})$ and $L_{\mathrm{FD}}=7.5 \mathrm{~mm}$. A compounded LF (III) has the same structural parameters used for the sub- elements (I) and (II). The ground plate sizes in structures (I), (II), and (III) are the same: $L_{x} \times L_{y}=30 \mathrm{~mm} \times 25.5 \mathrm{~mm}$.

The InvFLA is intended for installation in mobile equipment. In such a case, polarization purity (low cross polarization) is not required; however, an appropriate VSWR frequency response must be realized. The structural parameters for the InvFLA, Fig. 1(b), are obtained through the five steps described below, where the first three steps are rough adjustments and the fourth and fifth steps are devoted to a fine-tuning of the design.

For the first step, the inverted $L$ element [see inset (I) of Fig. 2] is analyzed for a height of $H_{L}=4.5 \mathrm{~mm}=0.0368 \lambda_{2.45}$, pre-selected in Section II, where $\lambda_{2.45}$ is the wavelength at $2.45 \mathrm{GHz}$ ( $\equiv f_{2.45}$ ). To obtain resonance around $f_{2.45}$, the horizontal length $L_{L}^{\prime}$ is chosen such that the total length $H_{L}+L_{L}^{\prime}$ is close to one-quarter wavelength at $f_{2.45}: L_{L}^{\prime}=27.5 \mathrm{~mm}\left(H_{L}+L_{L}^{\prime}=32 \mathrm{~mm}=0.261 \lambda_{2.45}\right)$. Resonance at $f_{2.45}$ is realized by adjusting the location of feed point (distance $L_{\mathrm{FD}}$ ). The VSWR frequency response for $L_{\mathrm{FD}}=7.5 \mathrm{~mm}$ is shown by the solid line in Fig. 2 .

The second step is performed using the inverted $F$ element shown in inset (II) of Fig. 2. The height $H_{F}$ is chosen to be smaller than $H_{L}$ for the inverted $L$ element, described in Section II: $H_{F}=2.5 \mathrm{~mm}=0.0433 \lambda_{5.2}$, where $\lambda_{5.2}$ is the wavelength at $5.2 \mathrm{GHz}\left(\equiv f_{5.2}\right)$. The feed point is located at the same point as that for the aforementioned inverted $L$ element $\left(L_{\mathrm{FD}}=\right.$ $7.5 \mathrm{~mm}$ ). Resonance around $f_{5.2}$ is obtained by choosing the horizontal length $L_{F}^{\prime}$ such that the $H_{F}+L_{F}^{\prime}$ is close to onequarter wavelength at $f_{5.2}: L_{F}^{\prime}=11 \mathrm{~mm}\left(H_{F}+L_{F}^{\prime}=\right.$ $13.5 \mathrm{~mm}=0.234 \lambda_{5.2}$ ). The VSWR for this structure is shown by the broken line in Fig. 2. Note that the horizontal lengths $L_{F}^{\prime}$ (obtained in the second step) and $L_{L}^{\prime}$ (obtained in the first step) are slightly changed to $L_{F}$ and $L_{L}$, respectively, after the fine-tuning in the fifth step.

The third step is to compound the inverted $L$ and $F$ elements determined in the first and second steps, as shown in inset (III) of Fig. 2. The white dots in Fig. 2 show the VSWR for this structure. It is observed that the VSWR at $f_{2.45}$ remains almost unchanged; however the VSWR at $f_{5.2}$ deteriorates due to mutual 


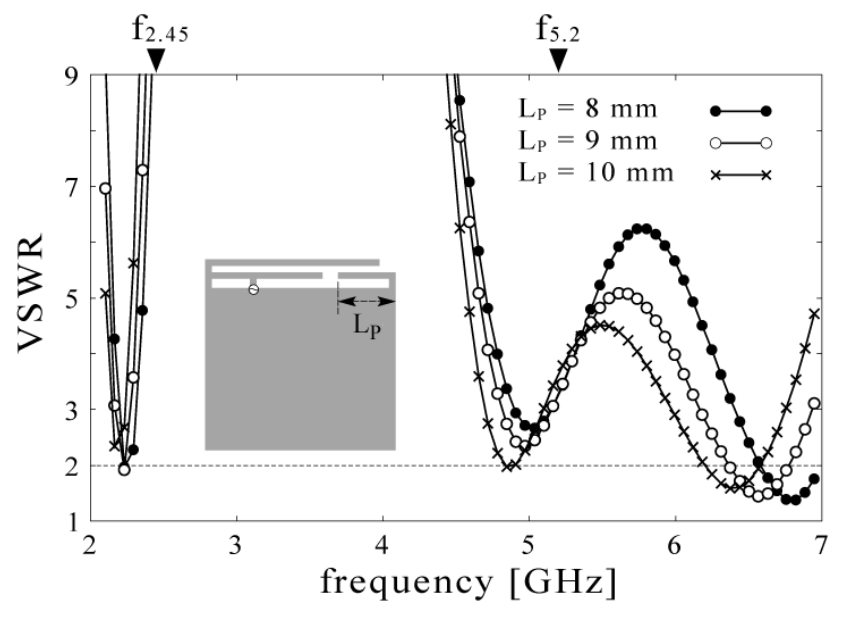

Fig. 3. Effects of the length of a parasitic $L$ element, $L_{P}$, on the VSWR, where the structural parameters $\left(H_{L}, L_{L}^{\prime}\right)=(4.5 \mathrm{~mm}, 27.5 \mathrm{~mm}),\left(H_{F}, L_{F}^{\prime}\right)=$ $(2.5 \mathrm{~mm}, 11 \mathrm{~mm})$, and $L_{\mathrm{FD}}=7.5 \mathrm{~mm}$ are used. The ground plate size is $L_{x} \times L_{y}=30 \mathrm{~mm} \times 25.5 \mathrm{~mm}$.

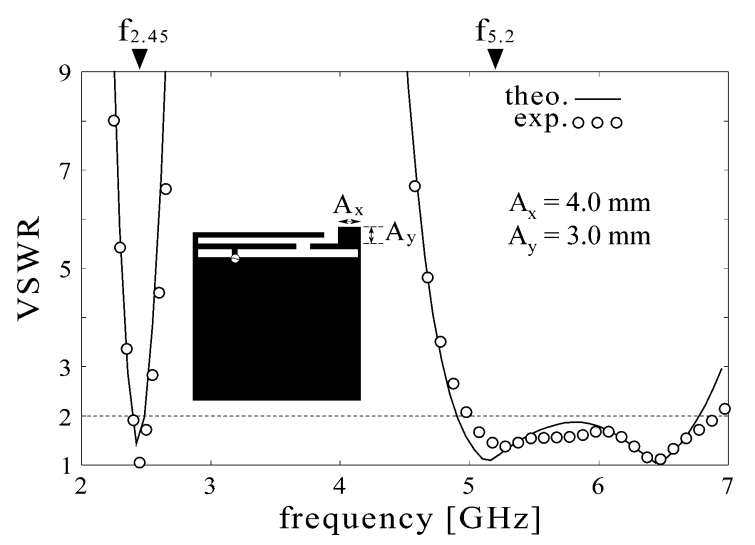

Fig. 4. VSWR for an inverted FL antenna. The structural parameters are $\left(H_{L}, L_{L}\right)=(4.5 \mathrm{~mm}, 24.5 \mathrm{~mm}),\left(H_{F}, L_{F}\right)=(2.5 \mathrm{~mm}, 10.5 \mathrm{~mm})$, $L_{\mathrm{FD}}=7.5 \mathrm{~mm},\left(H_{P}, L_{P}\right)=(2.5 \mathrm{~mm}, 9.0 \mathrm{~mm}),\left(A_{x}, A_{y}\right)=$ $(4.0 \mathrm{~mm}, 3.0 \mathrm{~mm})$, and $\left(L_{x} \times L_{y}\right)=(30 \mathrm{~mm} \times 25.5 \mathrm{~mm})$.

effects between the inverted $L$ and $F$ elements. This is overcome in the following fourth and fifth steps.

In the fourth step, a parasitic $L$ element is added to the structure discussed in the third step, as shown in Fig. 1(a). The height of the parasitic $L$ element is chosen to be equal to that of the inverted $F$ element, as described in Section II: $H_{P}=H_{F}=$ $2.5 \mathrm{~mm}$. Fig. 3 shows the VSWR as a function of frequency for three values of the horizontal length of the parasitic $L$ element, $L_{P}$, where the structural parameters for the inverted $L$ and $F$ elements are held at the values used in the third step: $\left(H_{L}, L_{L}^{\prime}\right)=$ $(4.5 \mathrm{~mm}, 27.5 \mathrm{~mm}),\left(H_{F}, L_{F}^{\prime}\right)=(2.5 \mathrm{~mm}, 11 \mathrm{~mm})$, and $L_{\mathrm{FD}}=7.5 \mathrm{~mm}$. It is found that the parasitic $L$ element generates resonances between 6 and $7 \mathrm{GHz}$. Note that the total length of the parasitic $L, H_{P}+L_{P}$, is close to one-quarter wavelength at the frequency where the minimum VSWR for each $L_{P}$ appears: $H_{P}+L_{P}=0.238 \lambda_{6.8}$ at $6.8 \mathrm{GHz}$ for $L_{P}=8 \mathrm{~mm}$, $H_{P}+L_{P}=0.253 \lambda_{6.6}$ at $6.6 \mathrm{GHz}$ for $L_{P}=9 \mathrm{~mm}$, and $H_{P}+L_{P}=0.267 \lambda_{6.4}$ at $6.4 \mathrm{GHz}$ for $L_{P}=10 \mathrm{~mm}$, where $\lambda_{f}$ is the wavelength at frequency $f$.


(a)

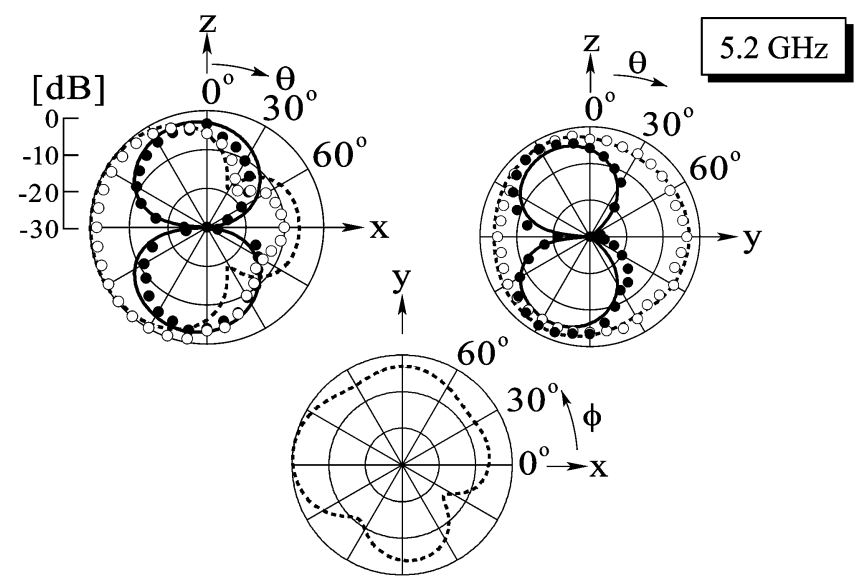

(b)

Fig. 5. Radiation patterns of an inverted FL antenna. (a) At $2.45 \mathrm{GHz}$. (b) At $5.2 \mathrm{GHz}$. The structural parameters are $\left(H_{L}, L_{L}\right)=(4.5 \mathrm{~mm}, 24.5 \mathrm{~mm})$, $\left(H_{F}, L_{F}\right)=(2.5 \mathrm{~mm}, 10.5 \mathrm{~mm}), L_{\mathrm{FD}}=7.5 \mathrm{~mm},\left(H_{P}, L_{P}\right)=$ $(2.5 \mathrm{~mm}, 9.0 \mathrm{~mm}),\left(A_{x}, A_{y}\right)=(4.0 \mathrm{~mm}, 3.0 \mathrm{~mm})$, and $\left(L_{x} \times L_{y}\right)=$ $(30 \mathrm{~mm} \times 25.5 \mathrm{~mm})$.

At this point there are two issues: 1) the VSWR curve is slightly shifted downward with respect to frequencies $f_{2.45}$ and $f_{5.2}$; and 2 ) the VSWR around $f_{5.2}$ is still larger than 2 . These issues are solved by the following structural modifications: 1) reduction of the original horizontal strip line lengths $L_{L}^{\prime}$ and $L_{F}^{\prime}$, and 2) widening of the strip width of the parasitic $L$ element. Note that the widening of the strip width is realized by making a protrusion on the parasitic $L$ element, where part of the strip line is widened to $w+A_{y}$ over length $A_{x}$, as shown in Fig. 1(b).

The fifth step is to perform the aforementioned structural modifications for dual-frequency operation at 2.45 and $5.2 \mathrm{GHz}$. Using trial and error, the structural parameters are determined to be $\left(L_{L}, L_{F}, L_{P}\right)=(24.5 \mathrm{~mm}, 10.5 \mathrm{~mm}, 9.0 \mathrm{~mm})$, 


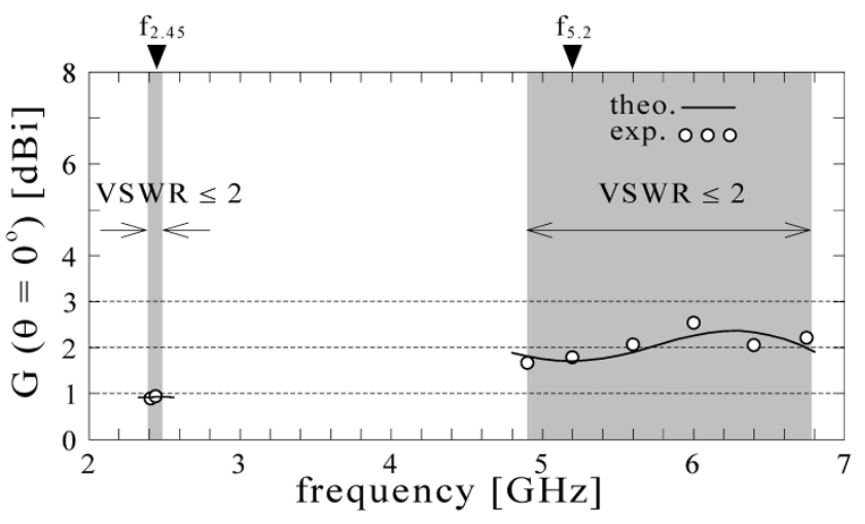

Fig. 6. Gain in the $z$-direction of an inverted FL antenna. The structural parameters are $\left(H_{L}, L_{L}\right)=(4.5 \mathrm{~mm}, 24.5 \mathrm{~mm}),\left(H_{F, L_{F}}\right)=$ $(2.5 \mathrm{~mm}, 10.5 \mathrm{~mm}), L_{\mathrm{FD}}=7.5 \mathrm{~mm},\left(H_{P}, L_{P}\right)=(2.5 \mathrm{~mm}, 9.0 \mathrm{~mm})$, $\left(A_{x}, A_{y}\right)=(4.0 \mathrm{~mm}, 3.0 \mathrm{~mm})$, and $\left(L_{x} \times L_{y}\right)=(30 \mathrm{~mm} \times 25.5 \mathrm{~mm})$.

$\left(A_{x}, A_{y}\right)=(4.0 \mathrm{~mm}, 3.0 \mathrm{~mm})$, and $L_{\mathrm{FD}}=7.5 \mathrm{~mm}$. Note that the horizontal lengths $L_{L}$ and $L_{F}$ are slightly smaller than the original lengths $L_{L}^{\prime}$ and $L_{F}^{\prime}$ in Fig. 1(a), respectively. Also note that the largest antenna height $\left(H_{P}+A_{y}\right)$ is very small with respect to the wavelength: $5.5 \mathrm{~mm}=0.045$ wavelength at $2.45 \mathrm{GHz}$. Fig. 4 shows the frequency response of the VSWR for the InvFLA defined with these final values for the structural parameters. This figure clearly indicates dual-frequency operation at $f_{2.45}$ and $f_{5.2}$. The frequency bandwidth for a VSWR $=2$ criterion is $4.1 \%$ for the $f_{2.45}$ band and $31.8 \%$ for the $f_{5.2}$ band. The results are confirmed by the experimental results (white dots).

Fig. 5 shows the radiation patterns at $f_{2.45}$ and $f_{5.2}$. For confirmation of the FDTDM results, experimental results in the principal planes ( $x-z$ and $y-z$ planes) are presented. Additionally, only the FDTDM results of the radiation patterns in the $x-y$ plane at $f_{2.45}$ and $f_{5.2}$ are presented for completeness. The radiation patterns are useful in understanding the gain characteristic in the $z$ direction $G\left(\theta=0^{\circ}\right)$, which is shown in Fig. 6 together with experimental results, where the shadowed areas in the figure show the VSWR bands. It is found that the gain (with respect to an isotropic source) is approximately $0.9 \mathrm{dBi}$ at $f_{2.45}$ and approximately $1.7 \mathrm{dBi}$ at $f_{5.2}$, with a small gain variation in each VSWR band. These gain values are small due to the fact that the radiation is not highly directive, as seen from the radiation pattern $E_{\phi}$.

The difference between the gains at $f_{2.45}$ and $f_{5.2}$ (i.e., the gain in the $z$-direction at $2.45 \mathrm{GHz}$ is smaller than that at 5.2 $\mathrm{GHz}$ ) is attributed to the following facts: 1) the radiation pattern $E_{\phi}$ at $2.45 \mathrm{GHz}$ in each of the $x-z$ and $y-z$ planes is more omnidirectional than that at $5.2 \mathrm{GHz}$ and 2) the radiation pattern $E_{\theta}$ at $2.45 \mathrm{GHz}$ in each of the $x-z$ and $y$ - $z$ planes (having a figure-eight pattern) shows a wider half-power beam width than that at $5.2 \mathrm{GHz}$.

An omnidirectional pattern is desirable for communications between a fixed base station antenna and an antenna installed in a mobile device. Note that, if a more omnidirectional $E_{\phi}$ pattern (in the $x-z$ plane) is required for the InvFLA at $5.2 \mathrm{GHz}$, this can be achieved by placing the parasitic $L$ element just under the horizontal strip line of the inverted $F$ [13].

\section{CONCLUSION}

An InvFLA, made of a thin conducting film, has a card-type structure, where the radiation element is a compound of inverted $L$ and $F$ elements, which is adjacent to a co-planar ground plate. The design procedure for dual-frequency operation at $f=$ $2.45 \mathrm{GHz}$ and $5.2 \mathrm{GHz}$ is described in five steps. In the first step, an inverted $L$ element is designed for operation at $2.45 \mathrm{GHz}$. In the second step, an inverted $F$ is designed for operation at $5.2 \mathrm{GHz}$. Based on these designs, a compound of the inverted $L$ and $F$ elements is investigated in the third step. Fine adjustment for dual-frequency operation is performed by introducing a parasitic $L$ element in the fourth step and then modifying the parasitic $L$ element in the fifth step.

It is found that the VSWR frequency bandwidth of the InvFLA is $4.1 \%$ around $2.45 \mathrm{GHz}$ and $31.8 \%$ around $5.2 \mathrm{GHz}$. It is also revealed that the $E_{\phi}$ component of the radiation field from the InvFLA spreads out in a somewhat omnidirectional fashion. Further analysis shows that the gain variation in each VSWR band is small. The gain in the $z$ direction (normal to the antenna plane) is $0.9 \mathrm{dBi}$ at $2.45 \mathrm{GHz}$ and $1.7 \mathrm{dBi}$ at $5.2 \mathrm{GHz}$.

\section{ACKNOWLEDGMENT}

The authors would like to thank V. Shkawrytko for his invaluable help in the preparation of this manuscript.

\section{REFERENCES}

[1] Y. Guo, M. Y. W. Chia, and Z. N. Chen, "Miniature built-in quad-band antennas for mobile handsets," IEEE Antennas Wireless Propag. Lett., vol. 2, pp. 30-32, 2003.

[2] M. Ali, G. J. Hayes, H. Hwang, and R. A. Sadler, "Design of multiband internal antenna for third generation mobile phone handsets," IEEE Trans. Antennas Propag., vol. 51, no. 7, pp. 1452-1461, Jul. 2003.

[3] Y. Guo, I. Ang, and M. Y. W. Chia, "Compact internal multiband antennas for mobile handsets," IEEE Antennas Wireless Propag. Lett., vol. 2, pp. 143-146, 2003.

[4] M. F. Abedin and M. Ali, "Modifying the ground plane and its effect on planar inverted- $F$ antennas (PIFA's) for mobile phone handsets," IEEE Antennas Wireless Propag. Lett., vol. 2, pp. 226-229, 2003.

[5] Y. Kwon, J. Moon, and S. Park, "An internal triple-band planar inverted-F antenna," IEEE Antennas Wireless Propag. Lett., vol. 2, pp. 341-344, 2003.

[6] D. Llorens, P. Otero, and C. C-Penalosa, "Dual-band, single CPW port, planar-slot antenna," IEEE Trans. Antennas Propag., vol. 51, no. 1, pp. 137-139, Jan. 2003.

[7] S. Yeh, K. Wong, T. Chiou, and S. Fang, "Dual-band planar inverted $F$ antenna for GSM/DCS mobile phones," IEEE Trans. Antennas Propag., vol. 51, no. 5, pp. 1124-1126, May 2003.

[8] H. Chen, W. Chen, Y. Cheng, and Y. Lin, "Dualband meander monopole antenna," in Proc. IEEE Antennas and Propagation Soc. Int. Symp., vol. 3, Columbus, OH, Jun. 2003, pp. 48-51.

[9] K. Wong, Compact and Broadband Microstrip Antennas. New York: Wiley, 2002

[10] A. Taflove, Computational Electrodynamics: The Finite-Difference Time Domain Method. Norwood, MA: Artech House, 1995.

[11] Z. P. Liao, H. L. Wong, B. P. Yang, and Y. F. Yuan, "A transmitting boundary for transient wave analysis," Science Sinica, Series A, vol. 27, no. 10, pp. 1063-1076, 1984.

[12] C. A. Balanis, Antenna Theory: Analysis and Design, 2nd ed: Wiley, 1982, pp. 575-594. 
[13] H. Nakano, K. Morishita, Y. Sato, H. Mimaki, and J. Yamauchi. A cardtype inverted LFL antenna for dual-frequency operation. presented at Proc. IEEE-ACES Int. Conf. Wireless Communications and Applied Computational Electromagnetics. [CD-ROM] s27p03i

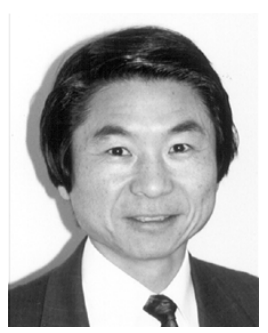

Hisamatsu Nakano (M'75-SM'87-F'92) was born in Ibaraki, Japan, on April 13, 1945. He received the B.E., M.E., and Dr.E. degrees in electrical engineering from Hosei University, Tokyo, Japan, in 1968,1970 , and 1974 , respectively.

Since 1973, he has been a Member of the Faculty of Hosei University, where he is now a Professor of Electronic Informatics. He was a Visiting Associate Professor from March to September 1981, at Syracuse University, Syracuse, NY. He was a Visiting Professor from March to September 1986, at the University of Manitoba, Canada, and from September 1986 to March 1987, at the University of California, Los Angeles. In 2001, he was appointed to the Guest Professorship of Shanghai Jiao Tong University, China. His research topics include numerical methods for low- and high-frequency antennas and optical waveguides. He has published more than 200 refereed journal papers, more than 180 international symposium papers, and more than 550 national symposium papers. He is the author of Helical and Spiral Antennas (New York: Research Studies Press, Wiley, 1987) and Helical and Spiral Antennas in Encyclopedia of Telecommunications (New York: Wiley, 2002) and is the coauthor of Analysis Methods of Electromagnetic Wave Problems, Volume Two (Norwood, MA: Artech House, 1996).

Prof. Nakano received the Institution of Electrical Engineers (IEE) International Conference on Antennas and Propagation Best Paper Award and the IEEE TRANSACTIONS ON ANTENNAS AND PROPAGATION Best Application Paper Award (H. A. Wheeler Award) in 1989 and 1994, respectively. In 1992, he was elected an IEEE fellow for contributions to the design of spiral and helical antennas. In 2001, he received the Award of Distinguished Technical Communication, from the Society for Technical Communication, USA. He is an Associate Editor of several journals and magazines, such as Electromagnetics, IEEE Antennas and Propagation Magazine, IEEE Antennas and Wireless Propagation Letters, and Asian Information-Science-Life.

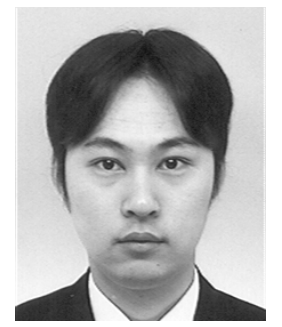

Yusuke Sato was born in Saitama, Japan, on August 14,1981 . He received the B.E. degree in electrical engineering from Hosei University, Tokyo, Japan, in 2004 , where he is currently working toward the M.E. degree.

Mr. Sato is a Member of the Institute of Electronics, Information and Communication Engineers (IEICE) of Japan.

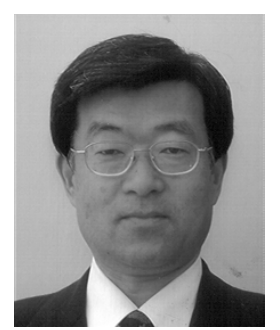

Hiroaki Mimaki (M'83) received the B.E. degree in electrical engineering from Tokyo Denki University, Tokyo, Japan, in 1976 and the M.E. degree in electrical engineering from Hosei University, Tokyo, Japan, in 1981.

$\mathrm{He}$ is currently an Assistant at Hosei University. His research interests are in thin wire antennas.

Mr. Mimaki is a Member of the Institute of Electronics, Information and Communication Engineers (IEICE) of Japan.

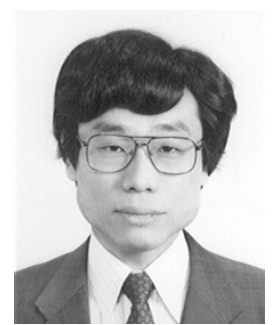

Junji Yamauchi (M'85) was born in Nagoya, Japan, on August 23, 1953. He received the B.E., M.E., and Dr.E. degrees from Hosei University, Tokyo, Japan, in 1976,1978 , and 1982, respectively.

From 1984 to 1988 , he served as a Lecturer in the Electrical Engineering Department of Tokyo Metropolitan Technical College. Since 1988, he has been a Member of the Faculty of Hosei University, where he is now a Professor of Electronic Informatics. He is the author of the book Propagating Beam Analysis of Optical Waveguides (Hertfordshire, U.K.: Research Studies Press, 2003). His research interests include optical waveguides and circularly polarized antennas.

Dr. Yamauchi is a Member of the Optical Society of America (OSA) and the Institute of Electronics, Information and Communication Engineers (IEICE) of Japan. 